\title{
Special issue on bilevel optimization
}

\section{Luce Brotcorne $^{1}$. Bernard Fortz ${ }^{1,2} \cdot$ Martine Labbé ${ }^{1,2}$}

Published online: 13 March 2020

(c) The Association of European Operational Research Societies and Springer-Verlag GmbH Berlin Heidelberg 2020

Bilevel programming is a fairly recent branch of optimization that deals with programs whose constraints embed an auxiliary optimization problem. Bilevel programs are pervasive and are commonly found in a number of real-world problems. This includes problems in the domains of transportation, energy, economics, decision science, environmental economics, security, etc.

Bilevel programming problems, being generically difficult to solve due to their nonconvexity and non-differentiability, it is not surprising that a large body of research to date has focused on problems having nice properties such as linear, quadratic or convex objective and/or constraint functions. Despite their apparent simplicity, these bilevel problems have been proved to be strongly NP-hard and even checking strict and local optimality is also NP-hard.

The research in bilevel programming can be classified into two parts: the design of algorithmic approaches to solve specific types of bilevel problems (e.g. linear or convex) and the definition of necessary and/or sufficient conditions of existence of equilibrium for more general bilevel programs.

In the last decade, one has observed a growing interest in bilevel programming, mainly due to its adequacy to model real situations involving competing agents.

Motivated by this intense development of the research domain, the first International Workshop on Bilevel Programming (IWOBIP) was organised in Monterrey, Mexico, in 2016. This workshop demonstrated the interest in gathering researchers from different optimization fields, game theory, and computer science but all presenting a central focus on bilevel optimization problems. As a consequence, the second edition of IWOBIP was organised in Lille, France, from 18 to 22 June 2018.

\footnotetext{
$\bowtie \quad$ Martine Labbé

mlabbe@ulb.ac.be

Luce Brotcorne

luce.brotcorne@inria.fr

Bernard Fortz

bfortz@ulb.ac.be

1 Inria Lille-Nord Europe, Avenue Halley 40, 59650 Villeneuve-d'Ascq, France

2 Computer Science Department, Université Libre de Bruxelles, CP 212, Boulevard du Triomphe, 1050 Brussels, Belgium
} 
This special issue on "Bilevel optimization" follows on IWOBIP 2018 but was not restricted to the participants of the conference. The papers address three different topics: polymatrix game, location, and support vector machines.

The first paper, "Bilevel programming methods for computing single-leader-multifollower equilibria in normal-form and polymatrix games" by Basilico et al., considers Stackelberg polymatrix games with followers playing a Nash equilibrium solution. Different cases are studied: pure or mixed strategies, optimistic, or pessimistic reaction of the followers. Single-level models when possible as well as black-box heuristics are proposed, and their performances are thoroughly evaluated.

The following two papers focus on competitive location problems. The paper "E-Constraint method for bi-objective competitive facility location problem with uncertain demand scenario" by Beresnev and Melnikov studies a Stackelberg game involving two firms locating facilities in order to maximize their respective profits. Customers patronize their most preferred open facilities, but the leader does not know precisely the set of customers. Hence, a probability distribution over a finite set of scenarii is provided. The authors propose a bilevel model with a first level involving two objectives corresponding to the maximisation of a minimum profit and its probability. Then, they develop an $\mathcal{E}$-approximate method to determine the set of efficient solutions.

The paper "Joint location and pricing within a user-optimized environment" by Dan et al. considers a firm maximizing its revenue by taking decisions regarding location, service levels, and prices. The second level of this problem is constituted by customers whose purchasing behaviour is influenced by congestion. The authors provide a nonlinear formulation with equilibrium constraints as well an efficient algorithm based on approximation to solve it to optimality.

The fourth and last paper of this special issue by Wei et al. concerns support vector machines that are widely used methods for supervised classification. Such methods contain a regularisation hyper-parameter to control the model complexity and overfitting. This paper proposes a bilevel optimization model to optimize this hyper-parameter. In the upper level, the hyper-parameter is optimized to minimise the prediction loss on validation data, while the lower level consists in the SVM problem itself on training data. The authors show the efficiency of a gradient-based method for solving this bilevel problem.

These four papers illustrate the adequacy of bilevel optimization for modelling and solving meaningful problems from very different areas. We are convinced that this interest for research in bilevel programming is only at its infancy, specially from its computational point of view.

Publisher's Note Springer Nature remains neutral with regard to jurisdictional claims in published maps and institutional affiliations. 